\title{
Intra-Pelvic Cup Migration as an immediate Post-Operative Complication
}

\author{
Francisco Alves*, André Spranger, Paulo Almeida, Joaquim Soares do Brito, Rita S Henriques and Alexandre \\ Fessenko
}

Hospital Santa Maria, CHLN, Portugal

*Corresponding author: Francisco Alves, Hospital Santa Maria, CHLN, Lisboa, Portugal

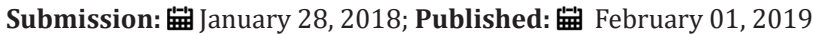

\begin{abstract}
Intra-pelvic cup migration is a rare but severe complication, mainly, due to the potential damage of vascular and other pelvic structures. This complication can result from several situations as asseptic loosening, dislocation, acetabular fracture and implant related failure. Safe intra-pelvic material removal has to be prepared to diminish the potential risk of damaging intra-pelvic structures.
\end{abstract}

Keywords: Hip protesis; Pelvic migration

\section{Case Presentation}

A 77-year-old female patient referred to our hospital due to an early postoperative complication: the intra-pelvic acetabular cup migration after primary hip arthroplasty. The patient had no relevant medical history. The $\mathrm{x}$-rays showed intrapelvic protusio of the prosthetic cup (Figure 1).

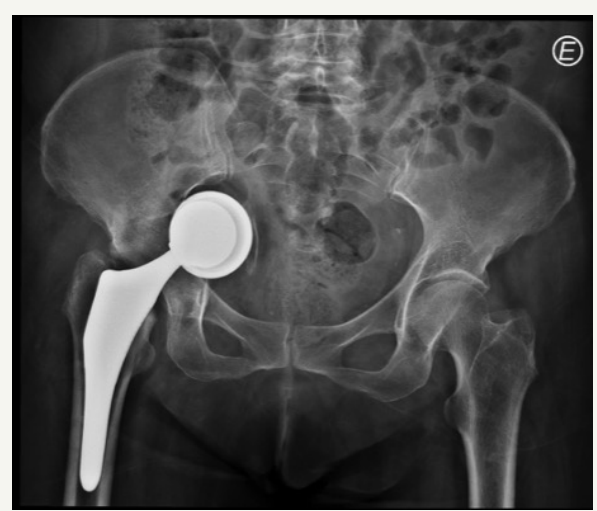

Figure 1: AP X- ray view.

The CT scan showed a bone defect and the proximity of prosthetic implants to the iliac vessels. There were no clinical or laboratorial signs of infection (Figure 2).

The surgical procedure was done in two stages. The first in supine postion: the Stoppa approach with the removal of the acetabular cup [1]. The second in lateral decubitus position: the Hardinge approach with the revision of the acetabular cup with a Bush-Schneider ring with a polyethylene cementation. As the femoral stem did not show signs of loosening, it was left in place and only the femoral head was changed. The intra operative maneuvers for assessing stability and abductor tension were correct. The post operative $\mathrm{x}$-ray showed no signs of failure. One week postoperatively the patient started weight bearing with progressive gait training (Figure 3); [2].

\section{Discussion}

In the setting of an intra-pelvic prosthetic migration careful assessment and planning are essential due to the relation it has with vital organs as the iliac vessels, bladder and the ureter. This type of complication is usually described in cases of bone deficiency associated with septic/aseptic loosening of the implants. Few reports exist about acute/subacute migration of hip prosthesis [3]. Our case represents an acute migration probably due to unrecognized intraoperative fracture of the acetabulum. The treatment of such lesions as the presented poses a great surgical challenge. Thus, preoperative planning is of paramount importance. A contrast enhanced helical CT scan is advisable, not only to evaluate the fractures lines but also to determine to relation of the migrated implants to the vascular structures.

The migrated acetabular cup can remain inaccessible through the conventional approach to the hip joint and the removal of the hardware can be difficult and dangerous [4]. The Stoppa approach, a retroperitoneal approach, offers a direct view of the prosthetic material allowing a safer withdrawal. The Stoppa approach is often used to repair acetabular fractures and as shown in this case can 
also be used in failed hip arthroplasty with intra-pelvic socket migration. This approach has some advantages: it allows the removal of the migrated cup with very good control of intrapelvic structures and it is less invasive than the transperitoneal approach.
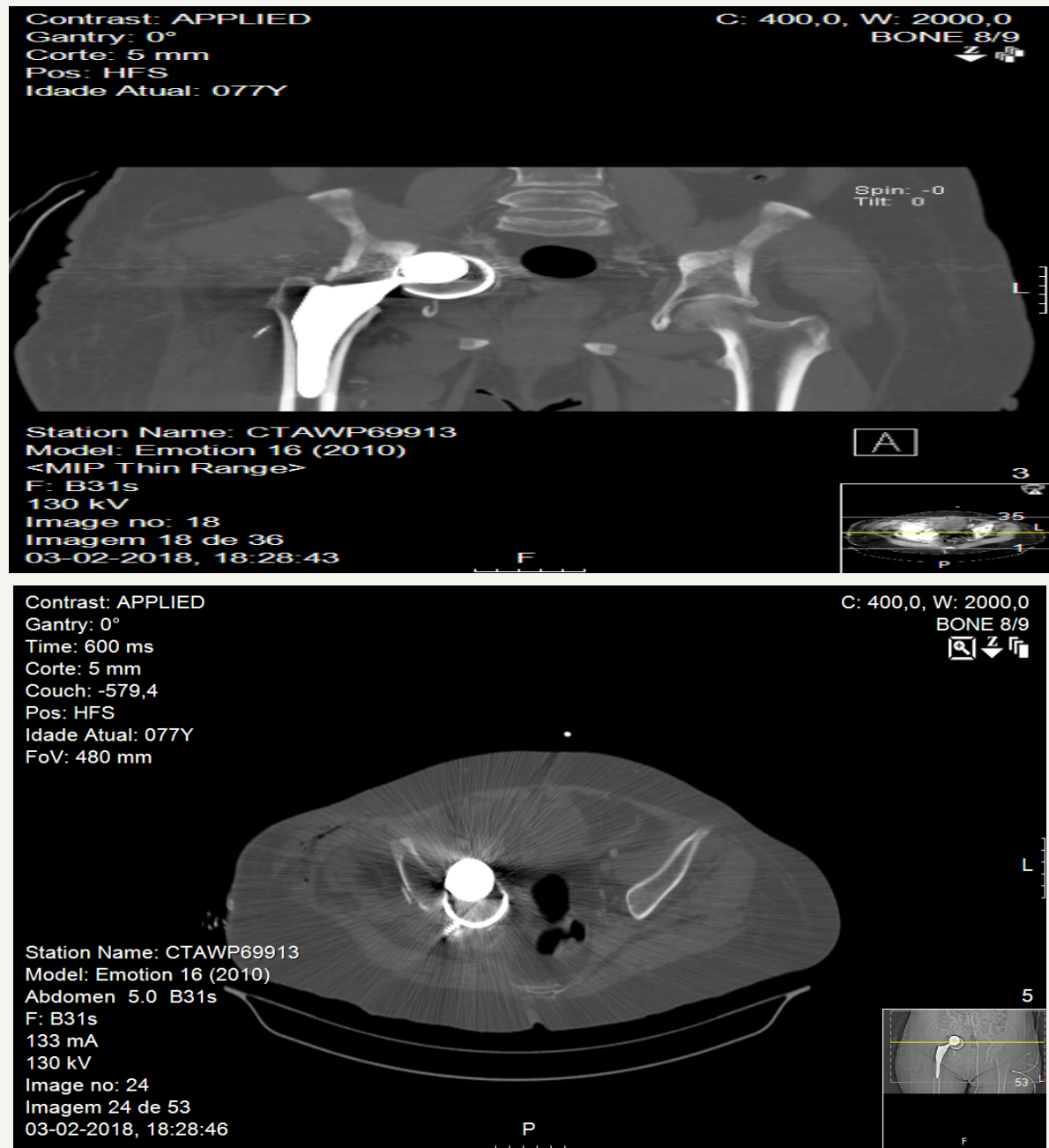

Figure 2: CT Scan views showing the intrapelvic prosthetic migration.

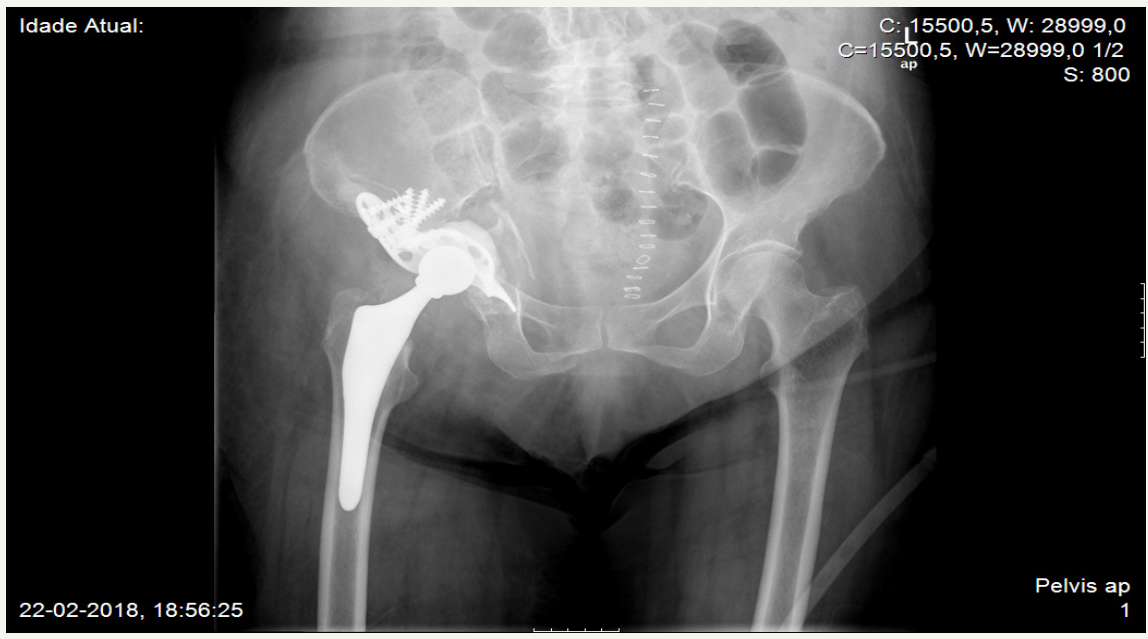

Figure 3: Postoperative X-ray. 


\section{References}

1. Girard AB, Wavreille G, Migaud H, Senneville E (2011) Total hip arthroplasty revision in case of intra-pelvic cup migration: designing a surgical strategy. Orthop Traumatol Surg Res 97(2): 191-200.

2. Hirvensalo E, Lindahl J, Kiljunen V (2007) Modified and new approaches for pelvic and acetabular surgery. Injury 38(4): 431-441.
3. Maeckelberg L, Simon JP, Naudie D, MacDonald S, Corten K (2012) Removal of an intra-pelvic socket: description of a safe surgical algorithm. Acta Orthop Belg 78(2): 152-158.

4. Barbier O, Pierret C, Bazile F, De Kerangal X, Duverger V, et al. (2012) Vascular complications following total hip arthroplasty: a case study and a review of the literature. Eur J Orthop Surg Traumatol 22(Suppl 1): $121-125$.

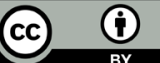

Creative Commons Attribution 4.0 International License

For possible submissions Click Here

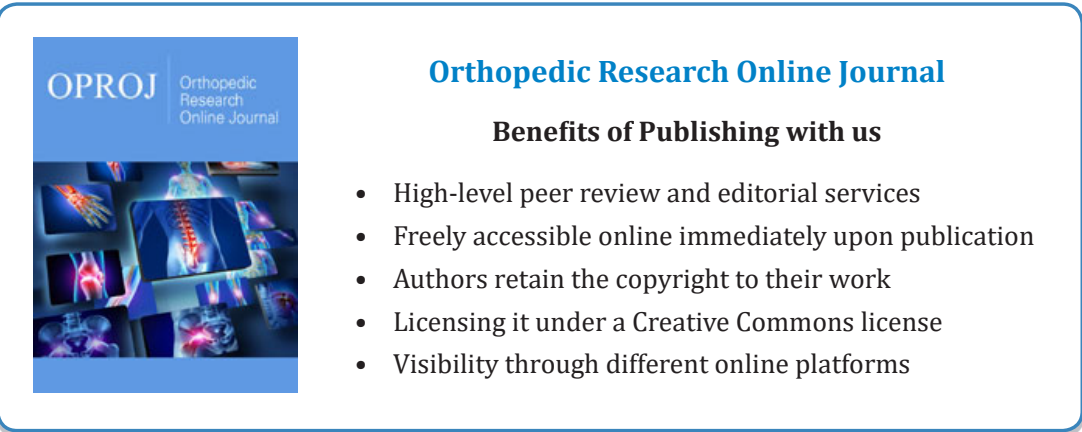

